\section{Avaliação de políticas públicas de segurança alimentar e combate à fome no período 1995-2002. 3 - O Programa Nacional de Controle da Deficiência de Vitamina A}

\author{
Evaluation of public policies to promote food \\ security and the fight against hunger, 1995-2002. \\ 3 - The Brazilian National Program to Control \\ Vitamin A Deficiency
}

\author{
Maisa Cruz Martins ${ }^{1}$ \\ Leonor Maria Pacheco Santos 2 \\ Sandra Maria Chaves dos Santos 3 \\ Maria da Purificação Nazaré Araújo 3 \\ Aline Maria Peixoto Lima 3,4 \\ Luciana Alaíde Alves Santana 5
}

\footnotetext{
${ }^{1}$ Agência Nacional de Saúde Suplementar, Ministério da Saúde, Rio de Janeiro, Brasil. 2 Departamento de Nutrição, Universidade de Brasília, Brasília, Brasil.

${ }^{3}$ Escola de Nutrição, Universidade Federal da Bahia, Salvador, Brasil. 4 Faculdade de Tecnologia e Ciência, Salvador, Brasil. 5 Departamento de Saúde, Universidade Estadual de Feira de Santana, Feira de Santana, Brasil.

Correspondência L.M. P. Santos Departamento de Nutrição, Universidade de Brasília. Campus Universitário Darcy Ribeiro, Brasília, DF 70910-900, Brasil. leopac@unb.br
}

\begin{abstract}
Vitamin A supplements have been distributed during National Immunization Days since 1983, and Brazil has been a pioneer in this kind of strategy. The current study evaluated the $\mathrm{Na}$ tional Program from 1995 to 2002, from a structure-process-results perspective. The methodology involved document research, interviews with health services managers, and a case study in 44 municipalities in Bahia State, resulting in 1,344 household interviews. In relation to structure, from 1998 on the program operated without a normative instrument due to the extinction of the National Institute of Nutrition. Improvements in the processes of vitamin supply procurement and distribution had a positive impact on coverage, reaching 3.5 million capsules in 2002 (72\% coverage). For the 2,546 children studied in Bahia, vitamin A distribution was irregular, with annual coverage varying from $8 \%$ to 26\%. Program managers reported time constraints and insufficient human resources, consistent with the hypothesis that this type of strategy does not help inform the population about the importance of vitamin A. These findings point to the need for systematic linkage between different levels of government in order to fully implement and monitor the program.
\end{abstract}

Food Security; Nutrition Programmes; Vitamin A

\section{Introdução}

As deficiências de micronutrientes são fatores de risco à saúde e sobrevivência de grupos vulneráveis, em especial os pré-escolares, sendo, em geral, causadas pela ingestão dietética insuficiente. Essas deficiências, por cursarem por vezes despercebidas, têm sido denominadas de "Fome Oculta" 1. A hipovitaminose A é uma das mais importantes deficiências nutricionais do mundo subdesenvolvido 1,2,3, afetando a população infantil no Brasil, em especial na Região Nordeste $3,4,5$.

A vitamina A, além de ser um micronutriente essencial para o funcionamento normal do sistema visual, está também envolvida na diferenciação celular, crescimento, reprodução e no sistema imune 3,4,5,6,7,8. Diversos estudos de intervenção com vitamina A demonstraram uma relação causa efeito entre a suplementação com esse micronutriente e a redução da taxa da mortalidade na infância e da severidade de doenças infecciosas. Por conta da associação entre a vitamina A e a morbi-mortalidade na infância, a Organização Mundial da Saúde (OMS), o Fundo das Nações Unidas para a Infância (UNICEF) e a Organização das Nações Unidas para a Alimentação e Agricultura (FAO) têm enfatizado a necessidade de serem instituídos programas de prevenção da deficiência desse micronutriente.

Na Reunião de Cúpula pela Infância, realizada em Nova York, em 1990, na Conferência pa- 
ra eliminar a Fome Oculta de 1991 em Montreal e na Conferência Internacional de Nutrição de 1992, em Roma, os chefes de Estado de países membros das Nações Unidas, inclusive o Brasil, assumiram o compromisso de "eliminação virtual da deficiência de vitamina A e suas conseqüências até o ano 2000" 1. Essa proposta incluía ações em longo prazo, que se sustentam nas estratégias de diversificação dietética e de saúde pública, e ações em curto prazo, representadas, especialmente, por programa de suplementação em massa com vitamina A, para as crianças sujeitas aos riscos da deficiência. A distribuição de megadoses de vitamina A, em dias nacionais de vacinação, tem ocorrido desde 1983, sendo o Brasil pioneiro nesse tipo de intervenção ${ }^{9}$. Atualmente, a OMS preconiza essa estratégia para tornar eficiente o combate à deficiência da vitamina A no curto prazo ${ }^{10}$, mas alerta sobre a necessidade da suplementação regular, duas vezes ao ano, para que os benefícios da vitamina A sobre a morbi-mortalidade na infância possam ser alcançados.

No Brasil, o reconhecimento da necessidade de combater a deficiência de vitamina A ganhou impulso nas últimas décadas. Existem levantamentos sobre a hipovitaminose A em crianças de 0 a 6 anos, em algumas regiões do Brasil, particularmente no Nordeste, desde a década de 80, com base em dados bioquímicos, em Pernambuco 6,11,12,13, Rio Grande do Norte 14, Ceará 6, Paraíba 13,15,16,17 e Bahia 18, em amostras não representativas de pré-escolares. Além disso, casos clínicos de xeroftalmia e cegueira nutricional foram registrados, entre 1981 e 1987, tanto na Paraíba 15,16,17 como no Rio Grande do Norte 14. Estudos realizados, após 1990, nos Estados de Pernambuco 19 e Sergipe 20 , em amostras representativas de préescolares, também fornecem informações sobre a deficiência de vitamina A. Esses estudos evidenciaram prevalências indicativas de problema de saúde pública nesses estados pelos critérios da OMS 4. Para os Estados do Piauí, Maranhão e Alagoas, não existem, até hoje, informações disponíveis sobre a hipovitaminose A.

Já nos anos 80, o Ministério da Saúde, através do Instituto Nacional de Alimentação e Nutrição (INAN - autarquia do Ministério da Saúde extinta em 1997) 21, concluiu que havia necessidade de ações em áreas consideradas de risco no país, após discussão e análise dos estudos existentes à época sobre a deficiência da vitamina A, apesar de esparsos. A intervenção posta em prática, a partir de 1983, foi a suplementação com cápsulas de vitamina A a crianças pré-escolares residentes em áreas de risco, associada à vacina antipólio em quase todos os Estados do Nordeste e no Vale do Jequitinhonha 9,22. Nesse período, algumas iniciativas de distribuição de vitamina A não partiram do Governo Federal, mas sim de Prefeituras Municipais (Ex.: Caruaru, Pernambuco e Feira de Santana, Bahia) e de ONG's, como a Cruz Vermelha (Ceará).

O Ministério da Saúde, em 1994, através da Portaria $n^{\circ}$. 2.160, instituiu o Programa Nacional de Controle das Deficiências de Vitamina A no âmbito do INAN 23 (PNVITA), englobando uma série de atividades de curto, médio e longo prazo. O programa foi instituído com a finalidade de contribuir para atender o compromisso assumido pelo Governo Brasileiro de erradicação da hipovitaminose A até o ano 2000. Após a publicação dessa Portaria, a distribuição de cápsulas de vitamina A foi intensificada, muito embora, a partir de 1998, o programa tenha operado sem uma estrutura normativa, com a revogação dessa Portaria, em decorrência da extinção do INAN.

Apesar de implantado há anos no Brasil, o programa nunca foi adequadamente avaliado. Pesquisa realizada, em 1986, demonstrou a efetividade desse tipo de suplementação no aumento dos níveis séricos de retinol 30 dias após a suplementação, efeito esse que se esgotou após 120 dias 9. Alguns aspectos operacionais e de custo foram analisados 24 , concluindo que o custo por criança beneficiada era cerca de U\$ 0,0100 na década de 80 . Mais recentemente, esse custo foi estimado em U\$ 0,0405 (dados internos da Coordenação Geral da Política de Alimentação e Nutrição para o ano de 2002).

O Estado da Bahia implantou o PNVITA em outubro de 1995, e não existem, até o momento, estudos de acompanhamento e avaliação. A preocupação da Secretaria da Saúde do Estado da Bahia (SESAB), no momento da implantação, centrou-se em possíveis efeitos colaterais da administração da vitamina, tanto que financiou um estudo para avaliar esse aspecto, cujo resultado demonstrou serem negligenciáveis os efeitos adversos 25 .

$\mathrm{O}$ estudo aqui apresentado faz parte de um projeto de pesquisa executado pela Escola de Nutrição, Universidade Federal da Bahia, que teve como objetivo avaliar os programas de segurança alimentar e combate à fome no Estado da Bahia, dentre os quais, o Programa Nacional de Combate às Deficiências da Vitamina A.

\section{Métodos}

A avaliação foi baseada no enfoque multidimensional alicerçado na tríade: estrutura-processoresultado 26 , já aplicada à avaliação do Programa de Alimentação do Trabalhador 27. Para a identificação das variáveis e construção dos instru- 
mentos de coleta de dados foi desenhada uma matriz específica para o PNVITA. Buscou-se avaliar o programa durante o período de 1995 a 2002, de acordo com as seguintes categorias de análise e investigação, em cada uma das dimensões do estudo.

\section{Dimensão de estrutura}

Instrumentos normativos; recursos humanos treinados; materiais disponíveis; estoque de cápsulas de vitamina A; competências organizacionais; critérios de seleção das áreas beneficiadas; infra-estrutura para monitoramento e controle do programa; material para divulgação do programa; entre outros.

\section{Dimensão de processo}

Estratégia de implantação do programa; processo de aquisição das cápsulas e fluxo do suprimento no tempo oportuno; sistema de transporte e distribuição do suplemento; implantação de estratégias educacionais e de sistema de monitoramento e acompanhamento.

\section{Dimensão de resultado}

Cobertura do programa nas áreas de risco e na população alvo; acesso ao programa em zona urbana e rural; focalização da implantação do programa; grau de conhecimento da população sobre o programa e sobre a importância da vitamina A.

Os dados utilizados para a análise do PNVITA foram obtidos a partir de pesquisa documental (dados oficiais do programa) e de planilhas de relatórios internos da SESAB sobre aplicação de megadoses de vitamina A no Estado. Foram também entrevistados gestores federais, estaduais e municipais, seguindo a metodologia do projeto principal 26.

A amostra do estudo de caso foi selecionada de modo intencional, para representar a população de mais baixa renda do Estado da Bahia. Dentre os 96 municípios inscritos no Programa Comunidade Solidária, 44 foram selecionados para a pesquisa. Foram entrevistados gestores municipais do PNVITA em 39 dos 42 municípios, nos quais o programa estava implantado.

Em cada município 40 famílias (20 urbanas e 20 rurais), residentes nos bairros e/ou povoados de mais baixa renda, foram selecionadas ao acaso. Nesses domicílios, todas as crianças que se encontravam na faixa etária do PNVITA foram identificadas (6 a 60 meses de idade em pelo menos um dos anos de acompanhamento, ou seja, de 1995 a 1998). O recebimento de doses de vi- tamina A nos anos de estudo foi anotado diretamente do Cartão da Criança, e foram aplicados questionários aos pais ou responsáveis. Esse processo resultou em uma amostra de 2.546 crianças, provenientes de 1.344 famílias. Vale ressaltar que o estudo principal incluía Salvador, a capital do Estado, porém o PNVITA não foi implantado nesse município por não ser considerado como área prioritária para essa carência pelo Ministério da Saúde.

\section{Resultados e discussão}

\section{Dimensão de estrutura}

A Portaria $n^{o} .2 .160$ foi o instrumento normativo que criou, no INAN, o PNVITA ${ }^{23}$. Nessa Portaria, constavam os seguintes objetivos:

- Garantir a eliminação da deficiência de vitamina A como um problema de saúde pública em áreas de risco no Brasil;

- Assegurar a suplementação com doses maciças de vitamina A em crianças de 6 a 59 meses de idade, residentes nas áreas de risco;

- Ampliar o conhecimento das famílias residentes em áreas de risco sobre a deficiência de vitamina A, visando ao aumento do consumo de alimentos ricos em vitamina $\mathrm{A}$;

- Desenvolver um processo de comunicação social para a popularização da importância da vitamina $\mathrm{A}$ e as conseqüências de sua deficiência;

- Estabelecer um sistema de monitoramento do programa que permita sua avaliação.

O programa deveria ser organizado em etapas bianuais de desenvolvimento, caracterizadas por progressivo deslocamento das prioridades de ação a partir das estratégias emergenciais até aquelas de caráter permanente, absorvidas pelas rotinas das instituições brasileiras. Assim, foi definido que os biênios 1995-1996 e 1997-1998 seriam as duas primeiras fases de implementação do programa; e 1999-2000, como fase de consolidação, na qual, paulatinamente, deveriam predominar ações permanentes nos serviços de saúde e as de maior alcance social. O programa foi planejado para cobrir o Nordeste e os municípios do Vale do Jequitinhonha, em Minas Gerais, regiões reconhecidas como "bolsões endêmicos" da deficiência de vitamina A.

Quanto à suplementação, essa seria feita pela distribuição de megadoses de vitamina A a cada seis meses, sendo empregadas cápsulas de 100.000UI para crianças de 6 a 11 meses de idade e de 200.000UI para crianças de 12 a 59 meses de idade. A estratégia recomendada pela Portaria foi distribuir o suplemento de vitamina A em par- 
ceria com o Programa Nacional de Imunização, por questões operacionais e econômicas, objetivando aumentar a cobertura. Porém, as normas do programa também abriram a possibilidade dessa distribuição ocorrer na rotina dos serviços de saúde.

Outra linha de ação proposta no programa foi desenvolver atividades de informação, educação e comunicação sobre a deficiência da vitamina A. Essa seria uma ação em longo prazo, objetivando o reconhecimento, pelas famílias em risco, dos problemas de saúde causados pela deficiência da vitamina A. Para maior efetividade foi sugerido que essas atividades deveriam ser precedidas de investigações etnográficas sobre o conhecimento, atitudes, crenças e práticas das mulheres residentes em áreas endêmicas sobre os alimentos regionais ricos em vitamina A. Materiais informativos e educativos regionalizados, enfocando alimentos ricos em vitamina A, seriam produzidos.

Outro componente, explicitado pela Portaria $n^{o}$. 2.160, foi o estudo descritivo do problema no país por meio de:

- Mapeamento de áreas de risco utilizando indicadores ecológicos e demográficos para áreas e populações de risco de deficiência de vitamina A;

- Confirmação biológica da área de risco, utilizando sorteio aleatório de agrupamento de riscos, efetuando-se o levantamento de indicadores biológicos de deficiência clínica ou subclínica de vitamina $\mathrm{A}$;

- Monitoramento das áreas de risco utilizando investigação anual dos indicadores biológicos da deficiência clínica e subclínica da vitamina A.

Atividades de monitoramento e avaliação seriam desenvolvidas por meio da análise periódica conjunta de três feixes de evidências relativas ao grau de funcionamento dos processos, aos efeitos biológicos e à eficácia da suplementação sobre a mortalidade e morbidade infantis.

As atividades de treinamento de recursos humanos seriam coordenadas pelo INAN, por meio da produção e distribuição do Manual de Normas Técnicas para Controle da Deficiência de Vitamina A, acompanhado por vídeo educativo a ser utilizado por monitores. Além dessas atividades, o INAN também deveria desenvolver estudos sobre a viabilidade técnica e econômica da fortificação de produtos de consumo de massa com vitamina $\mathrm{A}$.

A gestão do programa em nível federal era da responsabilidade técnica do INAN; após sua extinção, passou para a Coordenação Geral da Política de Alimentação e Nutrição do Ministério da Saúde. A gestão, em nível Estadual, ficaria a cargo das Secretarias Estaduais de Saúde, competindo a elas o repasse do treinamento sobre a aplicação das cápsulas de vitamina $\mathrm{A}$, a distribuição das cápsulas de vitamina A e a responsabilidade técnica para definir as estratégias de distribuição do suplemento. O treinamento da equipe local, a aplicação da vitamina A e o monitoramento da atividade cabiam ao município.

No decorrer deste estudo, em 1997, ocorreu a extinção do INAN, e, conseqüentemente, a Portaria no. 2.16023 foi automaticamente revogada. O programa continuou a existir, porém sem uma estrutura normativa, por quase oito anos. Somente em 13 de maio de 2005, o Ministério da Saúde publicou nova Portaria, a de número 729, instituindo o Programa Nacional de Suplementação de Vitamina A 28.

\section{Dimensão de processo}

Os gestores nacionais informaram que, durante quase duas décadas (1983-2000), a vitamina A era obtida por doação de instituições internacionais, como UNICEF, Sight and Life, Cruz Vermelha e Micronutrient Initiative. À época, cápsulas de vitamina A não eram fabricadas no Brasil. Não havia regularidade na entrega, provocando atraso na chegada aos municípios. Por vezes surgiam dificuldades na liberação do produto na alfândega, mesmo se tratando de doação de medicamentos. Visando melhorar a gestão do programa e garantir o provimento da vitamina, o Ministério da Saúde passou a adquirir cápsulas de vitamina A diretamente no Brasil, por intermédio da Fundação Oswaldo Cruz, a partir de 2001.

O processo de distribuição aos municípios cabia às Secretarias Estaduais de Saúde, responsáveis também pelo monitoramento do programa em nível local e pelo envio de relatórios periódicos ao Ministério da Saúde. Segundo informações dos gestores federais ouvidos pela pesquisa, eram constantes os atrasos no envio das planilhas consolidadas em nível central, em decorrência da espera dos dados enviados pelos municípios.

Seguindo os princípios do processo de descentralização previstos pelo Sistema Único de Saúde (SUS), a distribuição das megadoses para o público alvo cabia aos gestores municipais. As estratégias de distribuição mais freqüentemente utilizadas pelos municípios eram: (a) as Campanhas de Multivacinação; (b) a vacinação de rotina (c) o atendimento ambulatorial de pediatria e puericultura nas unidades de saúde e (d) os Agentes Comunitários de Saúde. Na maioria dos Estados do Nordeste, o dia de vacinação é a plataforma para distribuir vitamina A. Em 1998 esta estratégia era adotada em quarenta países, alcançando 60 milões de crianças 29. 
Segundo gestores federais entrevistados neste estudo as planilhas de acompanhamento do programa controlavam o número de doses distribuídas e não o número real de crianças suplementadas, porque crianças na faixa etária de 12 a 59 meses deveriam receber duas doses/ano. Para as crianças de 6 a 11 meses, o fato não chega a ser um problema, pois, nessa faixa etária, provavelmente, a criança só fará uso de uma dose no curso de seu primeiro ano de vida. Para efeito de análise dos dados nacionais nesta publicação, considerou-se que cada dose distribuída correspondia a uma criança beneficiada.

A dimensão de processo também foi avaliada no estudo de caso na Bahia. A SESAB iniciou a operacionalização do PNVITA em outubro de 1995, estabelecendo parcerias com o UNICEF, a Escola de Nutrição da Universidade Federal da Bahia e a Pastoral da Criança. A implantação do Programa e a sua expansão para os 415 municípios baianos, então existentes, seria feita de forma gradual, em decorrência de limitações estruturais e de recursos humanos e materiais. Foram poucos os municípios baianos que aderiram ao programa em 1995; na maioria dos casos, o programa só foi implantado no período de 1996 a 1997.

Segundo as informações obtidas dos gestores estaduais, a estratégia utilizada para implantação do PNVITA no Estado da Bahia foi a seguinte:

(a) Realização de treinamento com técnicos das Diretorias Regionais de Saúde (DIRES), sob responsabilidade da SESAB;

(b) Repasse do treinamento para técnicos dos municípios, sob responsabilidade das DIRES e SESAB;

(c) Implantação do programa nos municípios mediante estimativa do número de doses necessárias para cobertura da faixa etária alvo.

Durante o treinamento realizado com as DIRES, foi repassado o material explicativo sobre a técnica de aplicação das megadoses, fornecido pelo Ministério da Saúde (panfletos, manuais do instrutor, manual do aplicador, cartazes e cartilhas).

Segundo informações dos gestores estaduais o processo de suprimento das doses de vitamina iniciava com a estimativa anual do número de doses para cada município pela DIRES, de acordo com a quantidade de crianças atendidas em campanhas de imunização anteriores e a população na faixa etária beneficiada. A solicitação era encaminhada primeiramente em nível estadual (SESAB) e desse para o Ministério da Saúde. Na época do estudo de campo as doses eram doadas para o Governo Brasileiro que encaminhava aos Estados, cabendo à SESAB enviar para as DIRES e essas para os municípios com o programa implantado. O desenho do programa contemplava muitas possibilidades de ruptura, desde que dependia de um fluxo administrativo complexo entre as três esferas de governo.

Após a distribuição da vitamina cabia aos municípios repassar para as DIRES as informações quantitativas sobre número de doses aplicadas por faixa etária, anotadas em formulários próprios do programa, desenvolvidos pelo nível estadual. Por sua vez, as DIRES repassavam essas informações para a SESAB, que, por meio de relatório, encaminhava-as para o nível federal. O PNVITA foi implantado na Bahia em outubro de 1995, e não existem, até o momento, estudos de acompanhamento e avaliação, o mesmo ocorre nas demais Unidades da Federação.

O Cartão da Criança foi o instrumento utilizado para registro e controle da aplicação de vitamina A fornecida à criança. Inicialmente, essa anotação era feita à mão (caneta ou lápis), e, posteriormente, o Ministério da Saúde forneceu etiquetas próprias para registrar, no Cartão, a participação da criança no programa.

Em relação ao suporte técnico para implantação do programa nos municípios, foi observado o compromisso dos níveis federal e estadual no treinamento e suporte de material didático para os municípios, com exceção da fita de vídeo. Também foi evidenciada rotina de suprimento adequado das megadoses de vitamina $\mathrm{A}$, com exceção para o ano de 1998 quando ocorreram problemas de fornecimento, segundo informações internas da SESAB. Esse período de desarticulação do programa coincidiu com a extinção do INAN (1997-1998).

Dos 44 municípios visitados na Bahia, dois não tinham o programa implantado, e, em outros três municípios, não foi possível entrevistar os gestores do programa. Dos 39 gestores entrevistados, 36 (92\%) informaram que houve capacitação do técnico do município para aplicação do suplemento, e que o material didático (cartilhas, manuais e cartazes) foi recebido. Em média, foram treinados dois profissionais por município, geralmente os auxiliares de enfermagem que trabalhavam na rotina de vacinação. Apesar desse treinamento, apenas 9 dos 39 gestores municipais entrevistados (23\%) sabiam informar corretamente a faixa etária dos beneficiários do programa.

Quanto à forma de aquisição das cápsulas de vitamina $A$, todos relataram que o envio para o município era realizado através das DIRES. Por vezes, o município fazia a solicitação, e, outras vezes, o material era encaminhado mesmo sem a solicitação oficial. Não existia uma forma siste- 
mática de transporte das cápsulas de vitamina A para os municípios; na maioria das vezes, eram as DIRES que se responsabilizavam pelo envio, mas alguns municípios relataram que a prefeitura transportou o material.

Oito municípios referiram aplicar o suplemento de vitamina A apenas durante o Dia Nacional de Vacinação. A maioria optou por utilizar a aplicação nas campanhas de vacinação e também em procedimentos de rotina nas unidades de saúde. Alguns referiram aplicar em áreas específicas como urbana ou rural. A maioria dos municípios declarou encaminhar as planilhas de controle de aplicação de doses de vitamina A para as DIRES. Quanto à forma de registro no Cartão da Criança, 19 municípios relataram utilizar etiquetas específicas; 11, caneta e/ou etiqueta; e 9 , apenas caneta.

\section{Dimensão de resultado}

\section{- Cobertura nacional}

O número de doses de vitamina A aplicadas no Brasil e a cobertura nacional foram analisadas nas planilhas do Ministério da Saúde, obtidas a partir de dados (consolidados) fornecidos pelas Secretarias Estaduais de Saúde. Segundo esses dados, o número de doses foi de 1.553.232 no ano de 1995, 1.554.837 em 1996, aumentou para 1.864 .379 em 1998 e 2.479 .743 em 1999, mas caiu para 2.184.927 no ano 2000, 2.129.971 em 2001 e, finalmente, chegou a 3.551.008 em 2002 (para 1997 - dados não disponíveis). Melhorias nos processos de aquisição e distribuição da vitamina A impactaram positivamente na cobertura nacional, permitindo que, em 2002, fossem distribuídas 3,5 milhões de cápsulas. Esse incremento elevou a cobertura estimada da população alvo (crianças de 6 a 59 meses do nordeste e norte de Minas Gerais) de $28 \%$ para $72 \%$, em que pese a imprecisão na estimativa do denominador (população alvo: crianças pré-escolares). Contudo a irregularidade na oferta da vitamina se fez presente, e o aumento da cobertura não foi uma constante: no ano 2001, a cobertura registrada foi de 43,7\%, menor do que a de 1999 . Um panorama nacional do programa foi recentemente publicado por autores deste artigo 23 .

\section{- Cobertura e focalização na Bahia}

No caso da Bahia as planilhas anteriormente citadas informam que o número de doses foi de 202.280 no ano de 1995 (ano de implantação do programa), aumentou para 313.304 em 1996, alcançou 701.511 doses em 1999 (o dobro de 1996), mas caiu para 414.946 no ano 2000 e novamente para 212.517 em 2001, atingindo 352.642 doses em 2002. Para 1997 e 1998, as planilhas não informavam os dados da Bahia.

Todos os 44 municípios estudados se caracterizavam como "áreas de risco" para o PNVITA, por estarem localizados na região Nordeste e apresentarem grande vulnerabilidade social. Durante o período da pesquisa empírica (1995 a 1998), apenas dois dos 44 municípios estudados não haviam implantado o programa. Apesar disso, algumas crianças residentes nesses dois municípios receberam suplementos de vitamina A, provavelmente migrantes de outro local onde o programa estava implantado.

Das 2.546 crianças na faixa etária do PNVITA incluídas no estudo, 954 (37,5\%) receberam pelo menos uma dose de vitamina A em algum dos quatro anos que compreenderam a pesquisa ( $\mathrm{Fi}$ gura 1). Dessas, cerca de $90 \%$ receberam apenas uma dose ao ano, enquanto que o programa recomenda duas doses por ano. Esse resultado reflete a baixa cobertura do programa. Dentre aquelas suplementadas com vitamina A, apenas $49,7 \%$ dos responsáveis referiram terem sido informados de que a criança havia recebido vitamina A. Também foram encontradas 106 crianças $(4,1 \%)$ suplementadas fora da faixa etária preconizada pelo Ministério da Saúde (Figura 1).

Ressalta-se que a baixa cobertura do programa entre as crianças estudadas foi uma realidade durante todos os anos estudados. No ano de 1995, a cobertura observada foi de apenas $11 \%$, fato que poderia ser explicado pelo início de implantação do PNVITA na Bahia, que ocorreu em outubro de 1995. No decorrer, no ano de 1996, a cobertura observada aumentou para $23 \%$ e se manteve no mesmo patamar em 1997 (26,1\%). Em 1998, houve uma queda drástica nesse percentual passando para 8,8\% (Figura 2). Segundo informações da SESAB, por meio de relatório interno, a cobertura estadual para o programa, em 1995, foi de $63,5 \%$; em 1996, de 57,9\%.

As coberturas informadas pela SESAB contrastaram com os dados observados na pesquisa de campo. Talvez esse fato esteja relacionado ao não estabelecimento de meta baseada na população infantil, mas sim no potencial de cobertura do Estado. Em relação à informação obtida para o ano de 1997 (cobertura informada de 100\%), cumpre registrar que houve uma redução drástica na meta estabelecida pela SESAB, gerando aumento na cobertura calculada. Para o ano de 1998, foram relatados pelos gestores estaduais problemas de aquisição das cápsulas pelo Ministério da Saúde, acarretando atraso no envio para os Estados. Nota-se que, além da baixa cobertura, não existiu, nos municípios, uma distribuição contínua de megadoses de vitamina A pelo 
Cobertura global observada. Programa Nacional de Controle da Deficiência de Vitamina A, municípios do interior da Bahia, Brasil, 1995-1998.

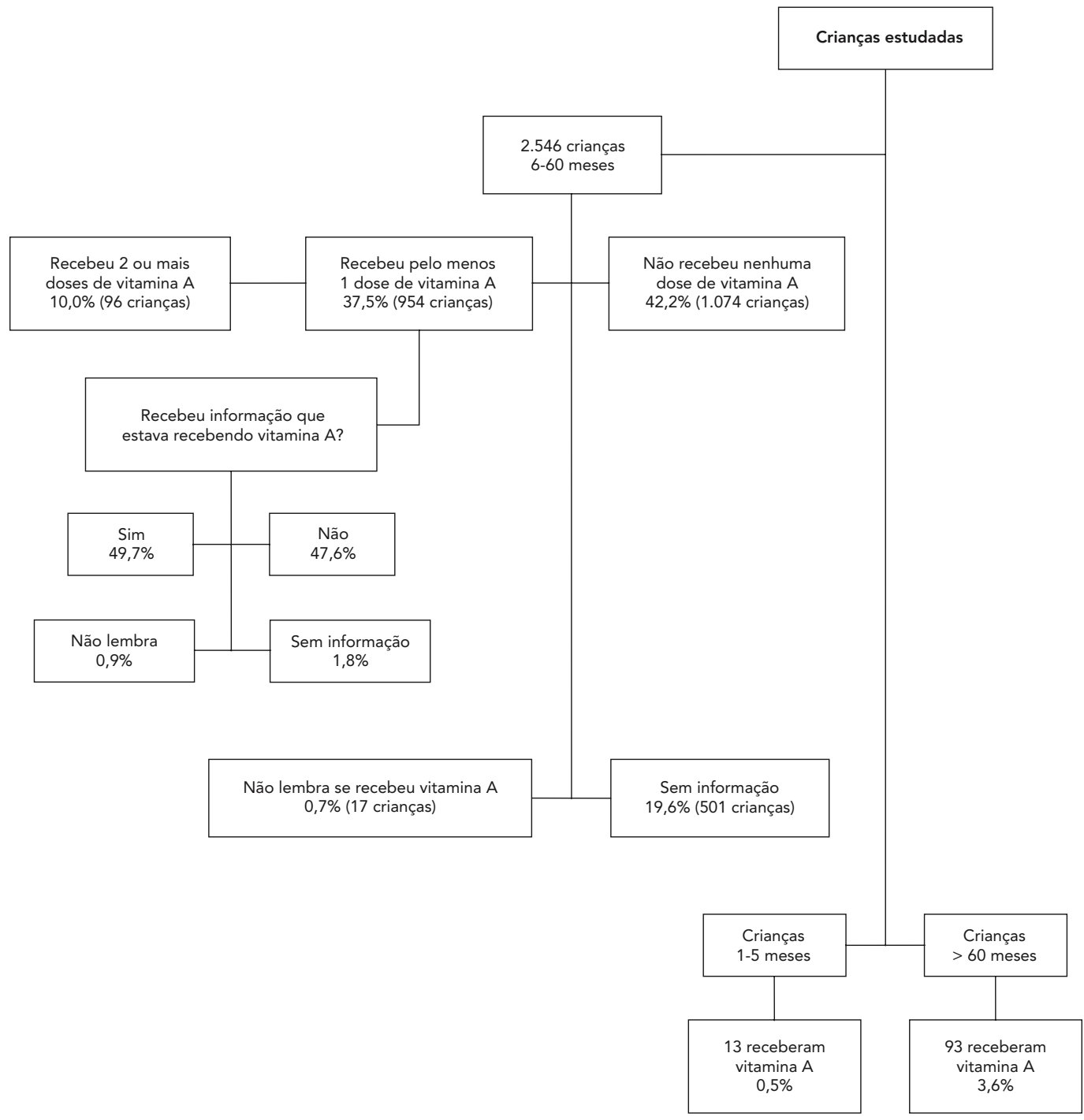

programa. Os valores de cobertura observados no estudo em campo foram muito mais baixos do que aqueles registrados em nível nacional, que foram de $28,2 \%$ (1995), 33,0\% (1996) e $42,4 \%$ (1998) (Figura 3).

Os dados do estudo de caso na Bahia mostraram que o acesso das crianças à suplementação com vitamina A não apresentou relação com a área de moradia. Proporções semelhantes foram observadas ao longo de três anos de estudo, com percentuais de cobertura observada urbano:ru- ral de 12,7:9,2 (1995)/22,5:23,5 (1996)/24,1:28,1 (1997). Apenas em 1998, a cobertura observada diferiu de modo significativo ( $p=0,001)$, sendo mais baixa na zona urbana $(6,1 \%)$ do que na rural $(11,3 \%)$.

Em relação à forma de registro da suplementação no Cartão da Criança, foi observado que nos anos de 1996 e 1997 predominou a marcação por meio de etiquetas, refletindo uma melhor organização do programa, contudo houve uma redução nessa utilização em 1998 (Figura 2). 
Figura 2

Cobertura anual observada. Programa Nacional de Controle da Deficiência de Vitamina A, municípios do interior da Bahia, Brasil, 1995-1998.

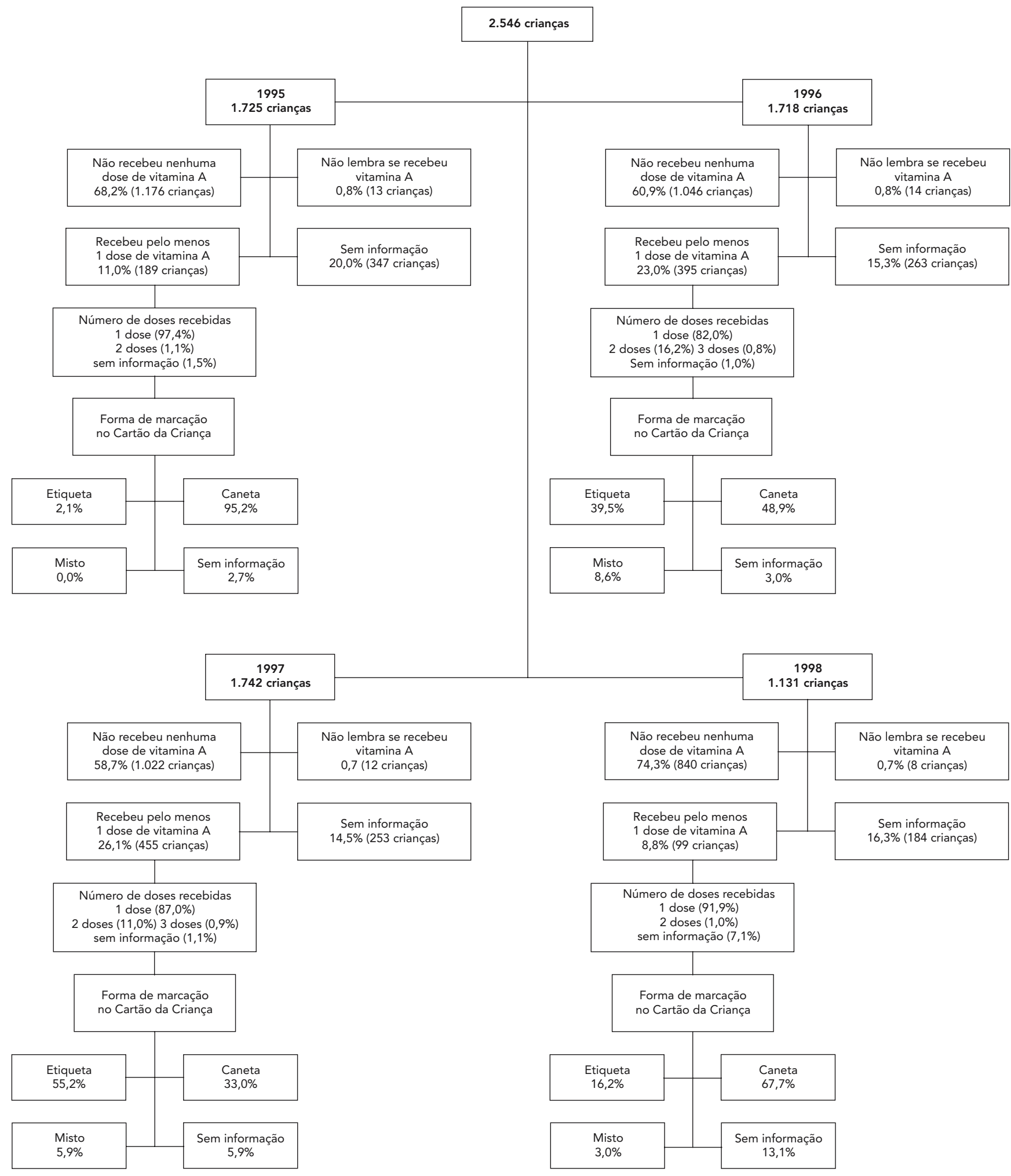


Número de doses e cobertura anual observada. Programa Nacional de Controle da Deficiência de Vitamina A, Brasil, 1995-2002.

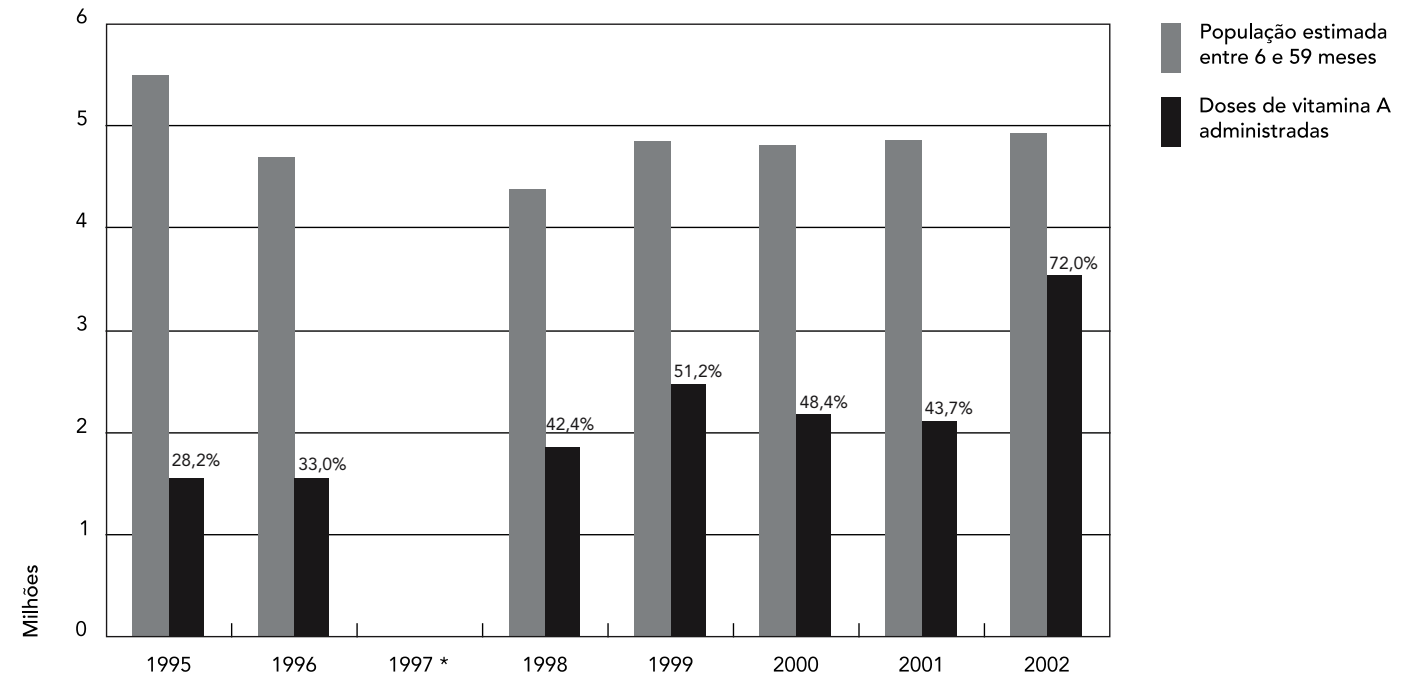

Fonte: Dados para 1995, 1996, 1999, 2002 disponíveis na Internet (http://www.saude.gov.br/nutricao);

Dados para 1998 (Goodman et al. 29).

* Dados para 1997 não disponíveis (período de extinção do Instituto Nacional de Alimentação e Nutrição).

Observou-se que do total de crianças que receberam pelo menos uma dose de vitamina (954), durante o período do estudo, 63,9\% provinham de famílias abaixo da linha de indigência; $16,7 \%$, abaixo da linha de pobreza; e $19,4 \%$ eram consideradas não pobres. É interessante notar que houve uma focalização adequada - maior cobertura nas famílias abaixo da linha de indigência. Porém, há que se considerar que a estrutura normativa do programa não preconiza focalização por nível sócio-econômico, mas sim por áreas de risco, nas quais a suplementação deve ser universal na faixa etária de seis meses a cinco anos.

\section{- Disseminação do conhecimento sobre vitamina $A$}

Quanto à avaliação do grau de entendimento das famílias beneficiadas sobre a vitamina A no Estado da Bahia, observou-se que a maioria (74,6\%) relatou não possuir nenhum tipo de conhecimento sobre vitamina A, e apenas $10,9 \%$ relataram terem obtido alguma vez informações sobre essa vitamina (Figura 4). Entre as famílias que obtiveram alguma informação sobre vitamina A, $131(57,3 \%)$ relataram que as informações foram obtidas nas unidades de saúde; e 15,3\%, nas campanhas de vacinação. Quando questionados sobre o tipo de informação obtida sobre a vitamina A, 47,3\% relataram que ela ajuda na nutrição da criança, mas alegações sem respaldo científico também foram obtidas, tais como combate à gripe (Figura 4) .

Dos 39 gestores municipais entrevistados, $32(82 \%)$ referiram conscientizar os pais ou responsáveis das crianças sobre a aplicação de megadose de vitamina $\mathrm{A}$, fato não comprovado pelas respostas obtidas nos questionários domiciliares. Entre esses gestores, apenas um referiu o desenvolvimento de material próprio (cartilha) para divulgação do programa, enquanto 18 declararam realizar algum tipo de disseminação de informações sobre a importância da vitamina $\mathrm{A}$ e/ou educação nutricional junto à comunidade.

A realização de atividades de informação, educação e comunicação sobre a deficiência da vitamina A seria um componente a longo prazo do PNVITA, objetivando ampliar o conhecimento, pelas famílias residentes em áreas de risco, sobre a deficiência de vitamina A e visando incentivar o aumento do consumo de alimentos ricos nessa vitamina. Os resultados encontrados 
Disseminação de conhecimento sobre vitamina A. Programa Nacional de Controle da Deficiência de Vitamina A, municípios do interior da Bahia, Brasil, 1995-1998.

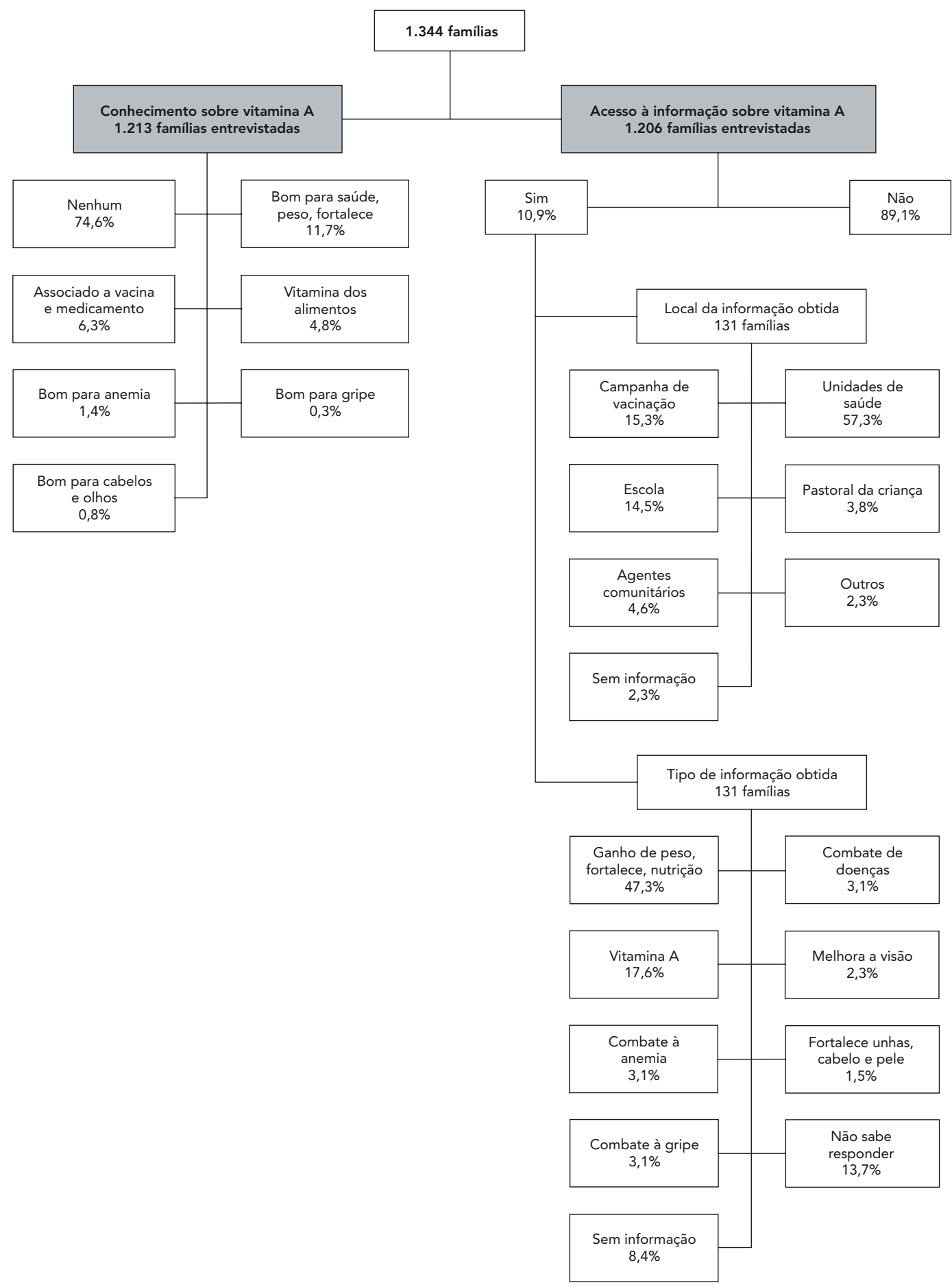


não condizem com os objetivos propostos, refletindo falha na divulgação sobre a importância dessa vitamina para a saúde das crianças, acarretando uma falta de conscientização das famílias sobre os benefícios da suplementação com vitamina A.

Limitações de recursos humanos e de tempo disponível para ações educativas no transcorrer dos dias nacionais de vacinação foram informadas pelos gestores. Observou-se que a estratégia recomendada pelo Ministério da Saúde - distribuir o suplemento por intermédio do Programa Nacional de Imunização - mostrou-se eficiente em relação ao número de doses aplicadas, contudo não foi eficaz no processo de repasse de informações, corroborando a hipótese de que esse tipo de estratégia não contribuiu para esclarecer a população sobre a importância da vitamina $\mathrm{A}$.

\section{Conclusões}

Apesar de contar com uma legislação apropriada em parte do período estudado (1995-1997), o programa operou sem estrutura normativa a partir de 1998, falha que só veio a ser corrigida em 2005. Já a infra-estrutura para montar e manter um sistema de monitoramento e controle do programa foi um dos pontos de estrangulamento. Melhorias nos processos de aquisição e distribuição da vitamina A impactaram positivamente, garantindo, a partir de 2001, o fluxo do suprimento da vitamina em tempo oportuno. No tocante aos resultados, houve incremento significativo no número de cápsulas distribuídas e na cobertura estimada em nível nacional.

Ressalta-se o não cumprimento de alguns componentes do programa, no que diz respeito ao mapeamento do problema no país, pois ainda não foram identificadas as áreas de risco, não foi realizada a confirmação biológica por meio de exames clínicos ou bioquímicos para detectar a deficiência subclínica de vitamina A, e, conseqüentemente, não foi possível implantar o monitoramento das áreas de risco por meio de investigação. No país, até a data atual, não existe um sistema nacional de vigilância epidemiológica sobre a hipovitaminose A, logo não é possível avaliar o impacto das medidas de prevenção através do programa a partir de dados gerados nos serviços de saúde.

Mesmo considerando os biênios 1995-1996 e 1997-1998 como fases iniciais de implementação do PNVITA, foram modestos os resultados obtidos na Bahia, nesse período, nos municípios estudados. A observação da baixa cobertura da suplementação com megadoses de vitamina A e a não implantação do programa em alguns municípios refletem falhas na dimensão de processo nos municípios estudados. Em virtude do desenho do programa, como antes destacado, essas falhas na implementação envolvem também as diretorias regionais, o estado e a união, pois contemplam muitas possibilidades de ruptura e dependem de um fluxo administrativo complexo entre as três esferas de governo.

Mesmo onde o programa estava implantado na Bahia, não existiu uma regularidade na distribuição do suplemento para as crianças. $\mathrm{O}$ Ministério da Saúde preconizava uma megadose de vitamina A a cada seis meses, porém foi observado que a maioria das crianças que foram suplementadas recebeu apenas uma dose ao ano. Não foram observados obstáculos para a suplementação em relação à área de residência da população alvo (urbana ou rural).

Em que pese algumas lacunas observadas, encontraram-se recursos humanos treinados e materiais informativos produzidos e distribuídos para a divulgação do programa na Bahia. As limitações de tempo e recursos humanos, informadas pelos gestores, corroboram a hipótese de que a estratégia de associar a distribuição da vitamina A com a vacinação não estaria contribuindo para esclarecer a população sobre a importância da vitamina A. Esse fato é evidenciado quando apenas uma pequena parcela dos entrevistados referiu ter obtido alguma informação sobre vitamina A durante a Campanha de Vacinação. A estratégia mais apropriada para desenvolver o processo de comunicação social para a popularização da importância da vitamina $\mathrm{A}$ e as conseqüências da sua deficiência seria o serviço de puericultura das unidades de saúde.

$\mathrm{O}$ recém criado Programa Nacional de $\mathrm{Su}$ plementação com Vitamina A 28 tem um longo caminho a percorrer no sentido de sua implementação adequada. Os achados deste estudo mostram que se faz necessária uma articulação sistemática entre as esferas de governo para que o programa seja implementado e monitorado em sua plenitude, com a regularidade desejada e de forma contínua. Como para outras políticas acredita-se como relevante que, desde a esfera federal, estratégias de indução sejam desenhadas, visando mobilizar e capacitar Estados e municípios para a ação e para o monitoramento e a avaliação do programa. 


\section{Resumo}

A distribuição de vitamina A em Dias Nacionais de Vacinação ocorre desde 1983; o Brasil foi pioneiro nesse tipo de intervenção. O estudo avaliou o programa no período de 1995-2002 na perspectiva de estruturaprocesso-resultado. A metodologia envolveu pesquisa documental, entrevistas com gestores e estudo de caso em 44 municípios da Bahia, resultando em 1.344 questionários domiciliares. Em relação à estrutura, o programa operou sem instrumento normativo a partir de 1998, decorrente da extinção do Instituto Nacional de Alimentação e Nutrição. Melhorias nos processos de aquisição e distribuição do suplemento impactaram positivamente na sua cobertura; em 2002, foram distribuídas 3,5 milhões de cápsulas (cobertura 72\%). Nas 2.546 crianças estudadas na Bahia, não houve regularidade na distribuição e a cobertura anual variou de 8\% a 26\%. As limitações de tempo e recursos humanos, informadas pelos gestores, corroboram a hipótese de que essa estratégia não contribui para esclarecer a população sobre a importância da vitamina. Os achados mostram ser necessária a articulação sistemática entre esferas de governo para que o programa seja executado e monitorado em sua plenitude.

Segurança Alimentar e Nutricional; Programas de Nutrição; Vitamina A

\section{Colaboradores}

M. C. Martins realizou a revisão da literatura e foi responsável pela análise dos dados aqui apresentados. L. M. P. Santos e S. M. C. Santos foram responsáveis pela concepção do projeto, elaboração dos instrumentos, coleta, análise e interpretação dos dados. M. C. Martins, M. P. N. Araújo, A. M. P. Lima e L. A. A. Santana participaram da elaboração dos instrumentos e da coleta de dados. Todos os autores são responsáveis pela autoria e revisão final deste texto.

\section{Agradecimentos}

Agradecemos a todos os sujeitos da pesquisa que aceitaram ser entrevistados. Além de reconhecer as longas jornadas de trabalho dos membros da nossa equipe, registramos, de modo especial, nossa gratidão ao Sr. Jorge F. B. Conceição, motorista da Universidade Federal da Bahia, que nos conduziu, em segurança, pelas estradas da Bahia.

Projeto financiado pela Financiadora de Estudos e Projetos (FINEP) com recursos do Banco Interamericano de Desenvolvimento (BID), processo no. 64.96.054900

\section{Referências}

1. Messner E. Conference report - Ending hidden hunger: A policy conference on micronutrient malnutrition. Food Nutr Bull 1992; 14(1). http://www.unu. edu/unupress/food/8F141e/8F141E00.htm.

2. Food and Agriculture Organization/World Health Organization. Final report of the International Conference on Nutrition. Rome: Food and Agriculture Organization/World Health Organization; 1993.

3. World Health Organization. Global prevalence of vitamin A deficiency: micronutrient deficiency information. Geneva: World Health Organization; 1995. (System Working Paper, 2).

4. World Health Organization. Indicators for assessing vitamin A deficiency and their application in monitoring and evaluating intervention programmes. Geneva: World Health Organization; 1996. (Micronutrient Series).
5. Diniz AS, Santos LMP. Hipovitaminose A e xeroftalmia. J Pediatr (Rio de J) 2000; 76 Suppl:S311-22.

6. McAuliffe J, Santos LMP, Diniz AS, Batista-Filho M, Barbosa RCC. A deficiência de vitamina A e estratégias para o seu controle: um guia para as $\mathrm{Se}$ cretarias Municipais de Saúde. Fortaleza: Project HOPE; 1991.

7. Underwood BA. Maternal vitamin A status and its importance in infancy and early childhood. Am J Clin Nutr 1994; 59:517-24.

8. Sub-Committee on Nutrition, Administrative Committee on Coordination, World Health Organization. 4th report on the world nutrition situation. Nutrition throughout the life cycle. Geneva: World Health Organization; 2000.

9. Santos LMP. Que falta faz a vitamina A? Ciênc Hoje 1986; 4:8-9. 
10. World Health Organization/International Vitamin A Consultative Group. Using immunization contacts to combat vitamin A deficiency. Report of an Informal Consultative Group of the World Health Organization. Geneva: World Health Organization; 1993.

11. Batista-Filho M, Torres MAA. Acesso à terra e situação nutricional em populações do semi-árido nordestino. Revista Pernambucana de Desenvolvimento 1982; 9:101-19.

12. Flores H, Araújo CRC. Liver levels of retinol in unselected necropsy specimens: a prevalence survey of vitamin A deficiency in Recife, Brazil. Am J Clin Nutr 1984; 40:146-52.

13. Batista-Filho M. Nutrição, alimentação e agricultura no Nordeste brasileiro: subsídios para uma política de extensão rural. 2a Ed. Brasília: Food and Agriculture Organization/Empresa Brasileira de Assistência Técnica e Extensão Rural; 1987.

14. Mariath JGR, Lima MCC, Santos LMP. Vitamin A activity of buriti (Mauritia vinifera Mart) and its effectiveness in the treatment and prevention of xerophthalmia. Am J Clin Nutr 1989; 49:849-53.

15. Dricot-d'Ans C, Dricot JM, Diniz AS, Mariath JGR, Santos LMP. Geographic distribution of xerophthalmia in the state of Paraiba, Northeast Brazil. Ecol Food Nutr 1988; 22:131-8.

16. Santos LMP, Dricot JM, Asciutti LS, Dricot-d'Ans C. Xerophthalmia in the state of Paraíba, Northeast Brazil: clinical findings. Am J Clin Nutr 1983; 38:139-44.

17. Santos LMP, Dricot JM, Asciutti LS, Benigna MJCN, Dricot-d'Ans C. Estudo epidemiológico da xeroftalmia no Estado da Paraíba. Rev Bras Med 1983; 40:419-25.

18. Santos LMP, Assis AMO, Martins MC, Araújo MPN, Morris SS, Barreto ML. Situação nutricional e alimentar de pré-escolares no semi-árido da Bahia (Brasil): II - Hipovitaminose A. Rev Saúde Pública 1996; 30:67-74.

19. Ministério da Saúde. II Pesquisa Estadual de Saúde e Nutrição: saúde, nutrição, alimentação e condições sócio-econômicas no Estado de Pernambuco. Recife: Instituto Nacional de Alimentação e Nutrição; 1998.

20. Martins MC, Santos LMP, Assis AMO. Prevalência da hipovitaminose A em pré-escolares no Estado de Sergipe. Rev Saúde Pública 2004; 38:537-42.
21. Brasil. Decreto no. 2.283, de 24 de julho de 1998. Dispõe sobre a extinção do Instituto Nacional de Alimentação e Nutrição - INAN e a desativação da Central de Medicamentos - CEME, e dá outras providências. Diário Oficial da União 1998; 25 jul.

22. Martins MC, Oliveira YP, Coitinho DC, Santos LMP. Panorama das ações de controle da deficiência de vitamina "A" no Brasil. Rev Nutr 2007; 20:5-18.

23. Brasil. Portaria ${ }^{\circ} .2 .160$, de 23 de dezembro de 1994. Cria, no Instituto Nacional de Alimentação e Nutrição, o Programa Nacional de Controle das Deficiências de Vitamina A e dá outras providências. Brasília: Diário Oficial da União 1994; 30 dez.

24. Santos LMP, Dricot-d'Ans C, Mariath GR, Dricot JM, Diniz AS. A preventive program for vitamin A defficiency in Paraiba, Northeast Brazil. In: Abstracts of the X Meeting of the International Vitamin A Consultative Group. Hyderabad: International Vitamin A Consultative Group; 1985.

25. Assis AMO, Santos LMP, Prado MS, Martins MC, Barreto ML. B. Tolerância à aplicação de megadoses de vitamina $\mathrm{A}$ associada à vacinação em crianças no Nordeste do Brasil. Cad Saúde Pública 2000; 16:51-7.

26. Santos LMP, Santos SMC. Avaliação de políticas públicas de segurança alimentar e combate à fome no período de 1995-2002. 1 - Abordagem metodológica. Cad Saúde Pública 2007; 23:1029-40.

27. Santos LMP, Araújo MPN, Martins MC, Veloso IS, Assunção MP, Santos SMC. Avaliação de políticas públicas de segurança alimentar e combate à fome no período 1995-2002. 2 - Programa de Alimentação do Trabalhador. Cad Saúde Pública 2007; 23:1931-45.

28. Brasil. Portaria n ${ }^{\circ} .729$, de 13 de maio de 2005. Institui o Programa Nacional de Suplementação de Vitamina A e dá outras providências. Brasília: Diário Oficial da União 2005; 16 mai.

29. Goodman T, Dalmya N, Benoist B, Schultink W. Polio as platform: using national immunization days to deliver vitamin A supplements. Bull World Health Organ 2000; 78:305-14.

Recebido em 23/Mar/2006

Versão final reapresentada em 10/Nov/2006

Aprovado em 23/Jan/2007 